\title{
An Overview of Direction-of-Arrival Estimation using an Antenna Array with Four Elements
}

\author{
Lotfi Osman, Imen Sfar and Ali Gharsallah \\ Department of Physics, UR-CSEHF, Faculty of Sciences of Tunis, \\ FST Campus Universitaire, El Manar University, 2092, Tunisia
}

Received 2012-04-20, Revised 2012-11-07; Accepted 2012-12-14

\begin{abstract}
The Direction-of-Arrival (DOA) estimation techniques using antenna arrays are applied in large areas of research fields and have received great attention in literature. Mobile communication, Sonar and Radar are just some examples among a large number of possible applications. For example, in military application, it is very important to recognize the direction of any threat. Another example of commercial application is to identify the direction of an emergency cell phone call so that the rescue unit can act in time to the right location. Thereby, DOA estimation using a traditional fixed antenna has many limitations and becomes inappropriate in such applications. In this letter, we describe a DOA estimation system based on an antenna array and five port circuits for detecting arrival angles in azimuth plane of signals pitched by the antenna array. For this, we have based on Multiple Signal Classification (MUSIC) algorithm, which showed a certain limit of its performance in spite of its big reputation by the researchers. In our case, we only consider uncorrelated signals and assume the channel parameters corresponding to a real context. The purpose is to compare the quality of the detected angle in term of precision depending on the number of the samples depicted. ADS software and MATLAB are used to achieve this study. The simulation results on the proposed system designed to operate at $2.4 \mathrm{GHz}$ frequency indicate that MUSIC algorithm is unable to automatically detect and localize the DOA of signals with high accuracy. Other algorithms exist and may provide more precision but require more computation time.
\end{abstract}

Keywords: Direction-of-Arrival (DOA), Advanced Design System (ADS), Local Oscillator (LO), Multiple Signal Classification (MUSIC)

\section{INTRODUCTION}

DOA estimation using a fixed antenna has many limitations (Godara, 1997). Its resolution is limited by the antenna's main lobe beam width. Antenna main lobe beam width is inversely proportional to its physical size. Improving the accuracy of angle measurement by increasing the physical aperture of the receiving antenna is not always a practical option. Some antennas especially used in military applications have physical size limitations. Consequently, they have comparatively wide main lobe beam width and the resolution cannot be good. Besides, if there are several signals falling down in the antenna main lobe, it will not be simple to distinguish between them. Instead of using a single antenna, an array antenna system with appropriate signal processing can improve the resolution of the received signals (Liao and Chan, 2011). An array sensor system has multiple elements distributed in space. The array configuration provides spatial samplings of the received waveform. A sensor array has better performance than the single sensor in signal reception and parameter estimation. Its high spatial resolution provides an efficient way to estimate the direction-of-arrival of multiple signals.

There are a variety of methods for the DOA estimation used including spectral estimation, minimum-

Corresponding Author: Lotfi Osman, Department of Physics, UR-CSEHF, Faculty of Sciences of Tunis, FST Campus Universitaire, El Manar University, 2092, Tunisia Tel: +216 71872600 Fax: +216 71871666 
variance distortion less response estimator, linear prediction, maximum entropy and maximum likelihood. In addition to previous methods, the most famous methods used in DOA are eigen structure methods, including many versions of MUSIC algorithms (Arja et al., 2009; Wang et al., 2010; Zahernia et al., 2011), minimum norm method (Gorodnitsky and Rao 1993) and ESPRIT method (Lavate et al., 2010).

We present in this study a complete DOA estimation procedure for three uncorrelated signals picked up by a uniform linear array antenna and by using MUSIC algorithm as high-resolution estimation method. We will show that in addition to the number of elements of the antenna array, the number of snapshots is one of the important parameters to take into consideration for better accuracy.

\section{MATERIALS AND METHODS}

The aim of this study is to present a system based on a uniform linear antenna array associated with five port circuits capable of measuring the DOA of RF (Radio Frequency) signals. The system is made of two main parts which are hardware part and software part. The hardware part is responsible of capturing RF signals and converting them in baseband. The software part detects the complex envelopes of signals and provides an estimation of the arrival angles.

The antenna array consists of four patch antennas operating at $2.4 \mathrm{GHz}$ frequency. The five-port circuits operate at the same frequency. We will describe in the following subsections these two essential elements of the DOA estimation system and will finish with a complete presentation of this device.

\subsection{Uniform Linear Array Antenna}

An array antenna is an essential part of a communication system (Anh and Cao, 2005). It can be used to exploit spatial and spectral characteristics of the incoming signals to provide highly accurate location information (Sfar et al., 2011).

Before implementing such system, a simulation step should be carried out in order to optimize its efficiency. In this study, we use an array antenna with a four element uniform linear array.

Figure 1 shows the general configuration for an array antenna having $M$ elements $(M=4$ in our case) arranged along a straight line with a distance d between sensor elements. The angle of the incoming signal $\theta_{\mathrm{N}}$ is determined relative to the antenna bore sight.

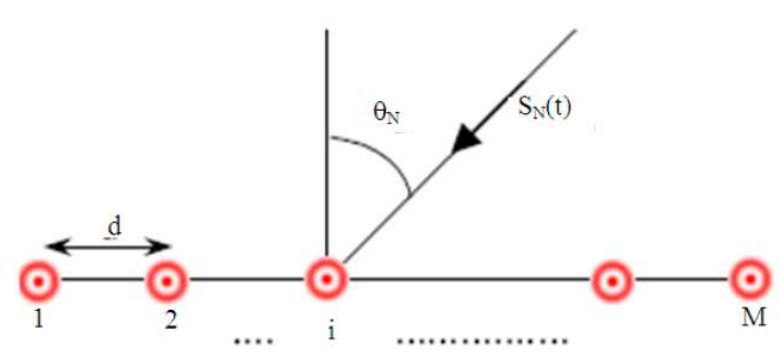

Fig. 1. Uniform linear array antenna configuration

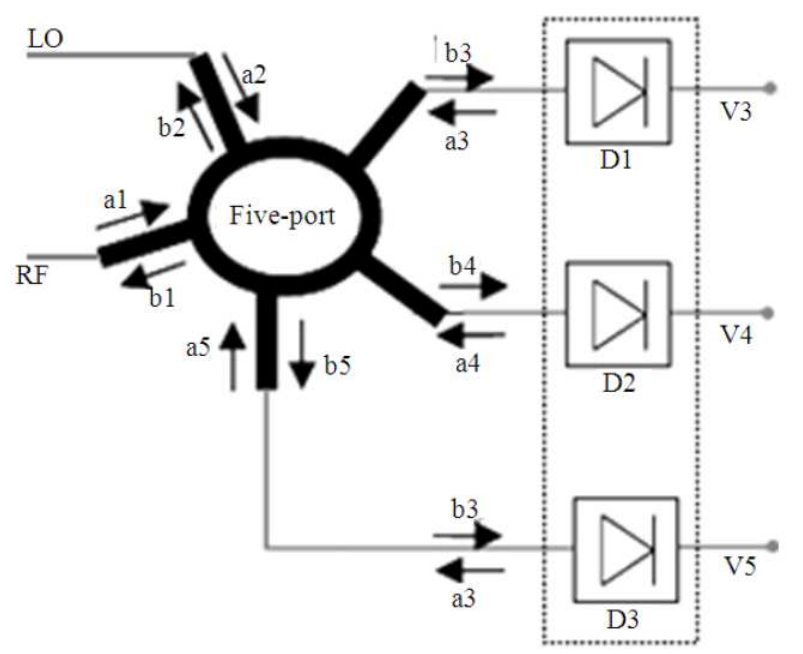

Fig. 2. Five-port circuit with three diode power detectors

\subsection{Five-Port Circuit}

The five-port technique has been recently applied to radio communications as a demodulator, to radars as a frequency discriminator and so on (Harabi et al., 2009; Mirzavand et al., 2010). Some advantages of the fiveport technique are its low cost and phase information which is obtained by making only amplitude or power measurement of three different linear combinations of $\mathrm{RF}$ and Local Oscillator (LO) electromagnetic waves (Sousa and Huyart, 2003).

The DOA is estimated by determining the phase difference of signals gathered by the antenna array and using a suitable high-resolution DOA estimation algorithm. As shown in Fig. 2, a five-port circuit is in fact a passive linear circuit with two inputs 1 and 2 excited by RF and LO signals, respectively.

The three outputs 3,4 and 5 are connected to three diode power detectors D1, D2 and D3 (Mouhamadou et al., 2006). 


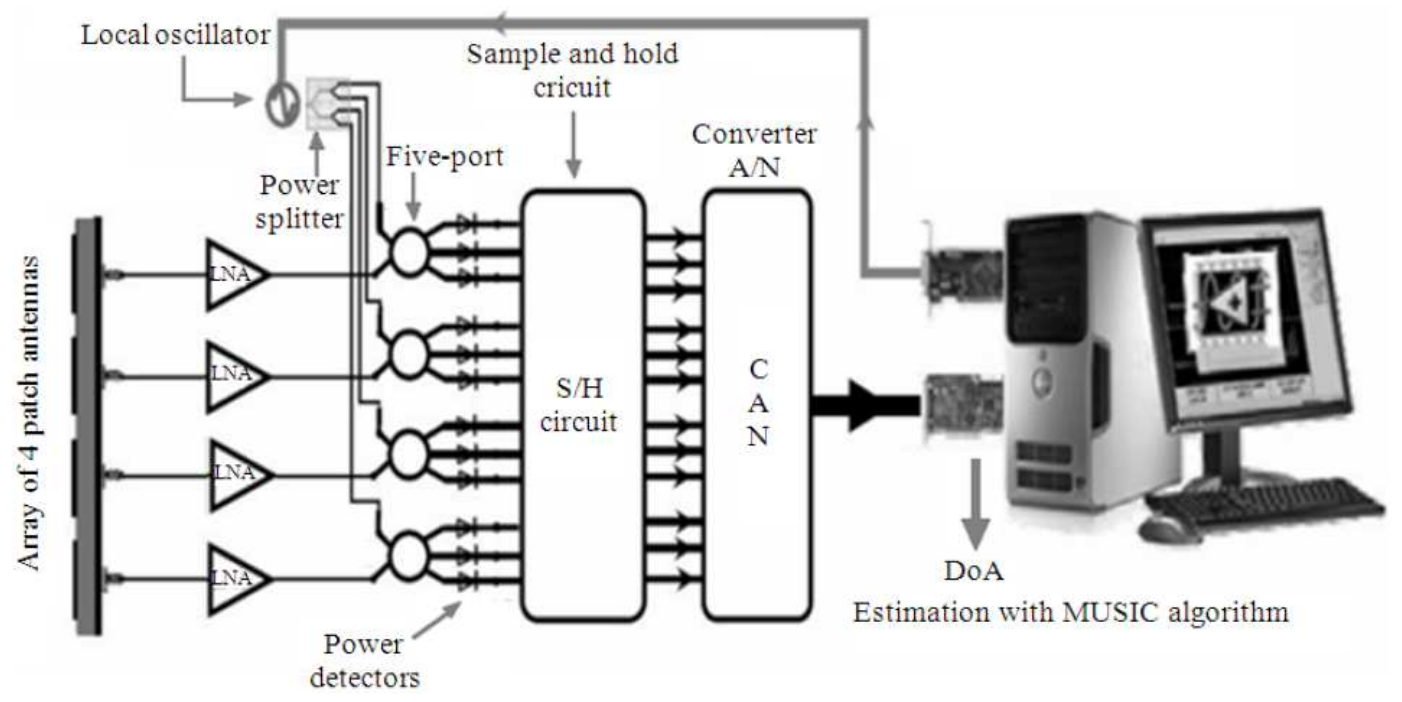

Fig. 3. Complete schematic of the DOA estimation system

\subsection{DOA Estimation System}

The complete system shown in Fig. 3 below includes mainly a four patch antennas array followed by four five-port circuits. These antennas are printed in FR4 substrate with $\varepsilon_{\mathrm{r}}=4.5$ and a board thickness equal to $1.59 \mathrm{~mm}$. The separation between consecutive elements is $\lambda / 2=43.25 \mathrm{~mm}$ in the horizontal axis, where $\lambda$ is the wavelength corresponding to $2.4 \mathrm{GHz}$ frequency. The dimensions of the patch antennas are optimized using Advanced Design System (ADS) software from Agilent Technologies to work around that frequency. The two inputs of each five-port are connected to the $\mathrm{LO}$ via a power splitter and one patch antenna through a Low Noise Amplifier (LNA), respectively. The five-port detector's outputs are connected to a Sample and Hold circuit $(\mathrm{S} / \mathrm{H})$ which is used to block the signals simultaneously before accomplishing an analog-to digital conversion through an acquisition card (Mouheddin and Jamel, 2009). Simultaneous acquisitions are very important for the phase discrimination of RF signals of the sensors, which is why the synchronization between the $\mathrm{S} / \mathrm{H}$ circuits is required.

The three output voltages at each five-port circuit are determined at all frequencies and stored for the post processing step. The ratio of RF and LO signals for each five-port is determined for all frequencies as a linear combination of the three voltages obtained at the detector's outputs (Sfar et al., 2011; Vu et al., 2005) Eq. 1:
$x(t)=I+j Q=g_{3} V_{3}+g_{4} V_{4}+g V_{5}$

where, $\mathrm{I}+\mathrm{jQ}$ is the complex envelop and $\mathrm{g}_{3}, \mathrm{~g}_{4}, \mathrm{~g}_{5}$ are the complex calibration constants.

\subsection{MUSIC Algorithm}

Multiple Signal Classification (MUSIC) is a relatively simple and efficient eigen structure method of DOA estimation. It has many variations and is perhaps the most studied method in its class (Khan et al., 2008).

The algorithm starts by assuming a linear array consisting of $\mathrm{N}$ antenna elements on which $\mathrm{M}$ signals impinge from directions $\emptyset_{1}, \emptyset_{2}, \ldots, \emptyset_{\mathrm{M}}$ such that $\mathrm{M}<\mathrm{N}$ (Quyen, 2005). At a particular instant of time $t=1,2, \ldots$, $\mathrm{K}$ where $\mathrm{K}$ is the total number of samples or snapshots taken for the incident signals, the array output which consists of signal and noise can be expressed as Eq. 2:

$\mathrm{u}(\mathrm{t})=\mathrm{x}(\mathrm{t})+\mathrm{n}(\mathrm{t})$

where, $x(t)$ and $n(t)$ are assumed to be uncorrelated. $n(t)$ is modeled as a temporally white and zero-mean complex Gaussian process. Signal vector $\mathrm{x}(\mathrm{t})$ is given by Eq. 3:

$\mathrm{x}(\mathrm{t})=\sum_{\mathrm{m}+1}^{\mathrm{M}} \mathrm{A}(\varnothing) \cdot \mathrm{S}(\mathrm{t})$

where, $\mathrm{S}(\mathrm{t})$ is an $\mathrm{M} \times \mathrm{l}$ vector of source waveforms and $A(\varnothing)$ an $N \times M$ matrix representing the array response to these sources (Al-Ardi et al., 2003; Peng et al., 2007). 
The matrix $A(\varnothing)$ contains the array steering vectors for the directions of the incident signals on the antenna array. By applying temporal averaging over $\mathrm{K}$ snapshots, the input covariance or correlation matrix $\mathrm{R}$ that builds up the data model can be expressed as Eq. 4:

$$
\mathrm{R}=\frac{1}{\mathrm{~K}} \sum_{\mathrm{t}=1}^{\mathrm{K}} \mathrm{u}(\mathrm{t}) \cdot \mathrm{u}(\mathrm{t})^{\mathrm{H}}
$$

The basic idea behind MUSIC algorithm is that the eigenvectors corresponding to the smallest eigen values (i.e., N-M eigen values) form the noise subspace and are also orthogonal to the steering vectors that make up the matrix A.

Therefore, by exploiting the orthogonality between the direction vectors and the noise subspace, the MUSIC angular or spatial estimate can be expressed as Eq. 5:

$$
\mathrm{P}(\theta)=\frac{\mathrm{A}(\varnothing)^{\mathrm{H}} \mathrm{A}(\varnothing)}{\mathrm{A}(\varnothing)^{\mathrm{H}} \mathrm{V}_{\mathrm{n}} \mathrm{V}_{\mathrm{n}}^{\mathrm{H}} \mathrm{A}(\varnothing)}
$$

where, $V_{n}$ is the matrix of eigen vectors corresponding to the noise subspace of the matrix $R$. Orthogonality between $A$ and $V_{n}$ minimize the denominator and hence give rise to peaks in the MUSIC spectrum. Those peaks correspond to the directions-of-arrival of the signals impinging on the antenna array. Another version of this algorithm, known as Root-MUSIC, has been proposed by Barabell (1983) to decrease its computational complexity. Root-MUSIC is based on polynomial rooting but it is applicable only in the case of uniform linear arrays.

\section{RESULTS}

As previously mentioned, the DOA is estimated by searching the phase difference of signals received by the antenna array with combination of a spectral estimation using MUSIC algorithm.

\section{DISCUSSION}

The numbers of snapshots and elements used are important parameters in the algorithm to ensure the accuracy of the direction-of-arrival of incident signals.

Three sources representing three transmitting antennas are considered in simulation. These sources represent three uncorrelated signals located at the DOA of 40,80 and $90^{\circ}$.

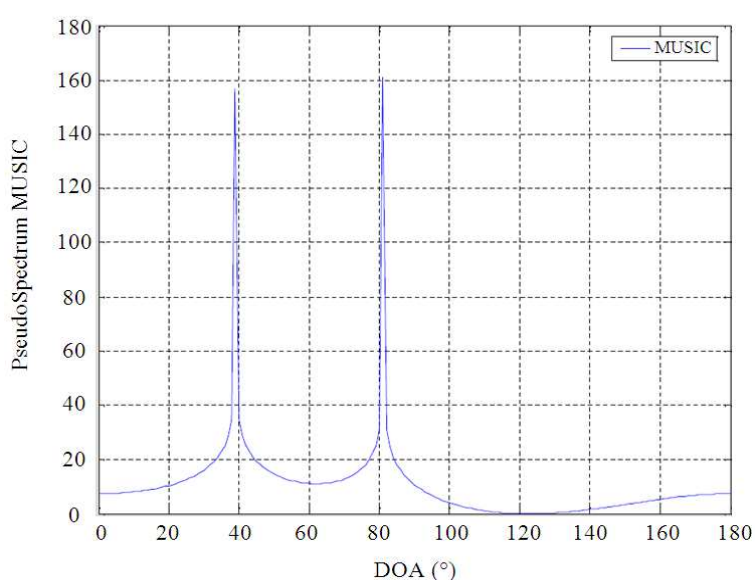

Fig. 4. Simulation result of three uncorrelated signals at the DOA of $40^{\circ}, 80^{\circ}$ and $90^{\circ}$ with 100 snapshots

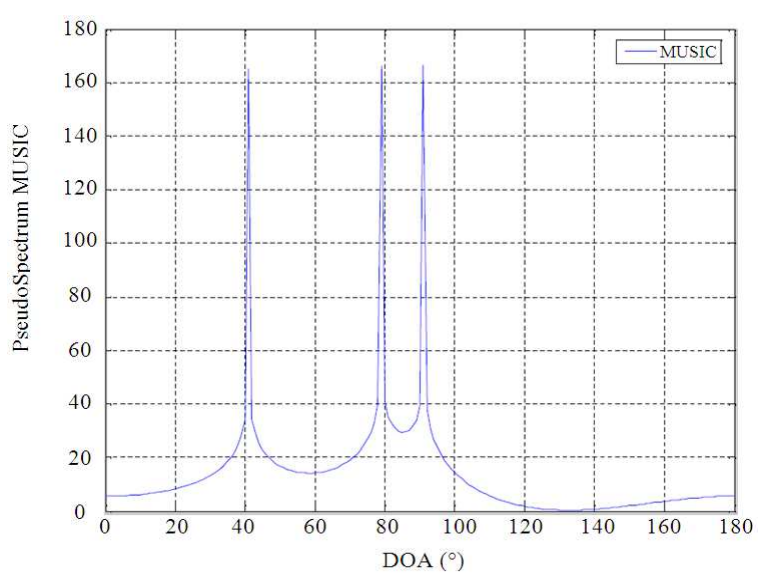

Fig. 5. Simulation result of three uncorrelated signals at the DOA of 40,80 and $90^{\circ}$ with 200 snapshots

In this study, we focus only on the number of snapshots. Simulations carried out with 100 and 200 snapshots are depicted in Fig. 4 and 5, respectively.

These figures show that this number is an important parameter to set carefully. As can be seen, the third angle $\left(90^{\circ}\right)$ does not appear in Fig. 4 because the number of snapshots is not large enough. It also shows that MUSIC algorithm is not always effective and consequently, it would be necessary to consider in certain cases other estimation techniques.

\section{CONCLUSION}

In this study, we have presented a system based on an array of patch antennas and five-port circuits to 
estimate direction-of-arrival of incoming signals. The high-resolution algorithm MUSIC associated with MATLAB program is used for DOA estimation. This technique is widely used by researchers (Vasylyshyn, 2007). As is reported in the literature and in spite of its robustness, MUSIC algorithm has some disadvantages such the number of antenna elements which must be bigger than the number of signals, or the number of snapshots which must be large enough as we have just shown it.

Compared to other techniques like ESPRIT (Estimation of Signal Parameters via Rotational Invariance Techniques) (Zhou et al., 2011), Maximum Likelihood (Tian and Huang, 2007) and Spectral Estimation Methods (Loadman et al., 2003), accurate results are relatively long to be produced when fewer elements are used in an antenna array. Anyway, these algorithms of estimation all available in literature have different characteristics and necessarily some limitations.

\section{REFERENCES}

1. Al-Ardi, E.M., R.M. Shubair and M.E. AlMualla, 2003. Investigation of high-resolution DOA estimation algorithms for optimal performance of smart antenna systems. Proceedings of the 4th International Conference on $3 \mathrm{G}$ Mobile Communication Technologies, Jun. 25-27, IEEE Xplore Press, pp: 460-464. DOI: $10.1049 /$ cp:20030417

2. Anh, P. and Q.T. Cao, 2005. DOA determination by using an antenna system without phase center and MUSIC algorithm. Proceedings of the IEEE Antennas and Propagation Society International Symposium, Jul. 3-8, IEEE Xplore Press, pp: 134-137. DOI: 10.1109/APS.2005.1552602

3. Arja, H.E., B. Huyart and X. Begaud, 2009. Joint TOA/DOA measurements for UWB indoor propagation channel using MUSIC algorithm. Proceedings of the 2nd European Wireless Technology Conference, Sept. 28-29, IEEE Xplore Press, Rome, pp: 124-127.

4. Barabell, A., 1983. Improving the resolution performance of eigenstructure-based directionfinding algorithms. Proceedings of the IEEE International Conference on Acoustics, Speech and Signal Processing, (ASSP' 83), IEEE Xplore Press, pp: 336-339. DOI: 10.1109/ICASSP.1983.1172124
5. Godara, L.C., 1997. Application of antenna arrays to mobile communications. II. Beamforming and direction-of-arrival considerations. Proc. IEEE, 85: 1195-1245. DOI: $10.1109 / 5.622504$

6. Gorodnitsky, I.F and B.D. Rao, 1993. A recursive weighted minimum norm algorithm: Analysis and applications. Proceedings of the IEEE International Conference on Acoustics, Speech and Signal Processing, Apr. 27-30, IEEE Xplore Press, Minneapolis, MN, USA., pp: 456-459. DOI: 10.1109/ICASSP.1993.319533

7. Harabi, F., I. Sfar and A. Gharsallah, 2009. A direction of arrival estimation system using five ports reflectometer. Proceedings of the 5th International Conference on Sciences of Electronic, Technologies of Information and Telecommunications, Mar. 22-26, IEEE, Tunisia, pp: 1-5. http://www.setit.rnu.tn/last edition/setit2009/Si gnal\%20Processing/134.pdf

8. Khan, Z.I., M.M. Kamal, N. Hamzah, K. Othman and N.I. Khan, 2008. Analysis of performance for Multiple Signal Classification (MUSIC) in estimating direction of arrival. Proceedings of the IEEE International RF and Microwave Conference, Dec. 2-4, IEEE Xplore Press, Kuala Lumpur, pp: 524-529. DOI: 10.1109/RFM.2008.4897465

9. Lavate, T.B., V.K. Kokate and A.M. Sapkal, 2010. Performance analysis of MUSIC and ESPRIT doa estimation algorithms for adaptive array smart antenna in mobile communication. Proceedings of the 2nd International Conference on Computer and Network Technology, Apr. 23-25, IEEE Xplore Press, Bangkok, pp: 308-311. DOI: 10.1109/ICCNT.2010.45

10. Liao, B. and S.C. Chan, 2011. DOA estimation of coherent signals for uniform linear arrays with mutual coupling. Proceedings of the IEEE International Symposium on Circuits and Systems, May 15-18, IEEE Xplore Press, Rio de Janeiro, Brazil, pp: 377-380. DOI: 10.1109/ISCAS.2011.5937581

11. Loadman, C., Z. Chen and D. Jorgensen, 2003. An overview of adaptive antenna technologies for wireless communications. Proceedings of the Communication Networks and Services Research Conference, May 15-16, Moncton, New Brunswick, Canada, pp: 15-19. 
http://www.cs.unb.ca/cnsrproject/Download/PD F/a3a.pdf

12. Mirzavand, R., A. Mohammadi and F.M. Ghannouchi, 2010. Five-port microwave receiver architectures and applications. IEEE Commun. Mag., 48: 30-36. DOI: 10.1109/MCOM.2010.5473861

13. Mouhamadou, M., G. Neveux and P. Vaudon, 2006. Simulation of smart antenna system using ADS Co-simulate with Matlab: Direction of arrival estimation and interference canceller. Proceedings of the IEEE Antennas and Propagation Society International Symposium, Jul. 9-14, IEEE Xplore Press, Albuquerque, New Mexico, pp: 4545-4548. DOI: 10.1109/APS.2006.1711648

14. Mouheddin, S. and B.H.T. Jamel, 2009. Indoor characterisation using high-resolution signal processing based on five-port techniques for signal input multiple output systems. Am. J. Eng. Applied Sci., 2: 365-371. DOI: 10.3844/ajeassp.2009.365.371

15. Peng, Y., Y. Feng and N. Zaiping, 2007. DOA estimation using music algorithm on a cylinderical conformal array. Proceedings of the IEEE Antennas and Propagation Society International Symposium, Jun. 9-15, IEEE Xplore Press, Honolulu, HI., pp: 5299-5302. DOI: 10.1109/APS.2007.4396743

16. Quyen, T.C., 2005. MUSIC for OFDM by using an uniform antenna array with two elements. Proceedings of the 1st Young Vietnamese Scientists Meeting, Jun. 12-16, Nha Trang, pp: $1-5$.

http://rts.gel.ulaval.ca/publications/uploadPDF/ publication_29.pdf

17. Sfar, I., L. Osman and A. Gharsallah, 2011. A five-port reflectometer for communication receiver applications. Proceedings of the 8th International Multi-Conference on Systems, Signals and Devices, Mar. 22-25, IEEE Xplore Press, Sousse, pp: 1-6. DOI: 10.1109/SSD.2011.5981483

18. Sousa, F.R.D. and B. Huyart, 2003. A new method for automatic calibration of 5-port reflectometers. Proceedings of the 2003 SBMO/IEEE MTT-S International Microwave and Optoelectronics Conference, Sept. 20-23, IEEE Xplore Press, pp: 1063-1068. DOI: 10.1109/IMOC.2003.1242869
19. Tian, L.W. and J.G. Huang, 2007. Maximum likelihood DOA estimator based on particle filtering. Proceedings of the International Conference on Machine Learning and Cybernetics, Aug. 19-22, IEEE Xplore Press, Hong Kong, pp: 2442-2445. DOI: 10.1109/ICMLC.2007.4370556

20. Vasylyshyn, V.I., 2007. Antenna array signal processing with high-resolution by modified beamspace music algorithm. Proceedings of the 6th International Conference on Antenna Theory and Techniques, Sept.17-21, IEEE Xplore Press, Sevastopol, pp: 455-457. DOI: 10.1109/ICATT.2007.4425243

21. Vu, V.Y., A.J Braga, X. Begaud and B. Huyart, 2005. Measurement of direction-of-arrival of coherent signals using five-port reflectometers and quasi-Yagi antennas. IEEE Microwave Wireless Components Lett., 15: 558-560. DOI: 10.1109/LMWC.2005.855391

22. Wang, P., G.J. Zhang, J.J. Xiong, C.Y. Xue and W.D. Zhang, 2010. Root-MUSIC algorithm with real-valued eigendecomposition for acoustic vector sensor array. Proceedings of the IEEE 1st International Conference on Pervasive Computing, Signal Processing and Applications, Sept. 17-19, IEEE Xplore Press, Harbin, China, pp: 652-656. DOI: 10.1109/PCSPA.2010.163

23. Zahernia, A., M.J. Dehghan and R. Javidan, 2011. MUSIC algorithm for DOA estimation using MIMO arrays. Proceedings of the 6th IEEE International Conference on Telecommunication Systems, Services and Applications, Oct. 20-21, IEEE Xplore Press, Bali, Indonesia, pp: 149-153. DOI: 10.1109/TSSA.2011.6095424

24. Zhou, L., D. Huang, H. Duan and Y. Chen, 2011. A modified ESPRIT algorithm based on a new SVD method for coherent signals. Proceedings of the IEEE International Conference on Information and Automation, Jun. 6-8, IEEE Xplore Press, Shenzhen, pp: 7578. DOI: 10.1109/ICINFA.2011.5948966 